\title{
Analysis of Various Textural Descriptors for Ovarian Cyst Classification
}

\author{
Sheela.S a,c,1, Sumathi.M ${ }^{\mathrm{b}}$, Sumathy.S ${ }^{\mathrm{c}}$, Thirumoorthy.S ${ }^{\mathrm{c}}$, Subalakshmi.E ${ }^{\mathrm{c}}$ \\ ${ }^{a}$ Research Scholar, Sathyabama Institute of Science and Technology \\ ${ }^{b}$ Professor, Sathyabama Institute of Science and Technology \\ ${ }^{c}$ Department of ECE , Rajalakshmi Institute of Technology, India
}

\begin{abstract}
Ovarian cyst is one of the main causes of infertility. Ovarian cancers are also caused by the ovarian cyst that grows in the ovary. An ovarian cyst can be benign (noncancerous) or malignant (cancerous). If the cyst is not diagnosed and treated at the earliest, the benign cyst may turn into malignant and can be fatal. Various image processing techniques are used to assist the clinicians to characterize the ovarian cyst using the textural descriptor. This paper reviews several textural descriptors for feature extraction like Local Binary Pattern (LBP), Local Directional Pattern (LDP) and Local Optimal Oriented Pattern (LOOP). Finally, extracted features are applied to SVM, KNN and Ensemble classifiers to compare the performance of the textural descriptors.
\end{abstract}

Keywords: Ovarian Cyst, Ovarian Cancer, Textural Descriptor, Local Binary Pattern, and Classification.

\section{Introduction}

The extraction of features from the ultrasound images is a very critical task in the area of medical image processing. Initially, input images are preprocessed to improve the quality of the image and region of interest (ROI) is segmented manually. Subsequently, feature extraction methods are employed to obtain the features that will be useful for image classification. The main purpose of the feature extraction is to get the related information from the image in low dimensional space. If the input image is large enough to process and is suspicious of redundancy, the input image is converted to a reduced set of features which is known as feature extraction (Kumar, 2014). The two types of features are gernal features and domain-specific features. Gernal features include shape, color, and texture (Bala, 2017). In this, the texture is a feature that provides information about the spatial arrangements of intensity or colors in the image. Textures are categorized by the spatial distribution of adjacent intensity levels. Collecting features by texture analysis is termed as texture feature extraction. In this paper, the effort has been taken in such a way that section I reviews various types of descriptors used to extract the texture features. Section II describes the overall process involved in the classification of the ovarian cyst as a simple (non-cancerous) or cancerous cyst. Section III evaluates the performance of the descriptors. Finally, this 
paper ends with the conclusive comment that the local binary pattern is the best texture feature extraction technique for the ovarian cyst classification.

\section{Texture Descriptors}

The texture is repetitive pattern information or layout of the structure at regular intervals. At all meanings, texture refers to the surface characteristic and the appearance of the object is given by the size, density, arrangement, etc.(Bala, 2017). The elementary step to gathering these features through the texture analysis process is known as texture feature extraction.

The most widely used textural descriptors are:

- Local Binary Pattern (LBP)

- Local Directional Pattern (LDP)

- Local Optimal Oriented Pattern (LOOP)

\subsection{Local Binary Pattern (LBP)}

The LBP descriptor (Ojala et al., 1996) serves as the local spatial pattern identifier and the grayscale difference. The LBP tags the binary quantity for each pixel and is assigned by using each bit distinct a pixel of a known span. Figure 1(a) illustrates a simplified model that tags every pixel is a variety that is acquired from its three by three neighbors. If the pixel is larger than the middle pixel, the binary value is fixed to 1 , and vice versa. The 8-bit binary model is formed where it consists of bits and its decimal value that emerge as the labels for middle pixel, displayed in a circle. Extensible to LBP encoder allows the neighbors of different sizes (Ojala et al., 2002). Round symmetry by defining parameter pairs $(\mathrm{P}, \mathrm{R})$, neighbors can be generated:

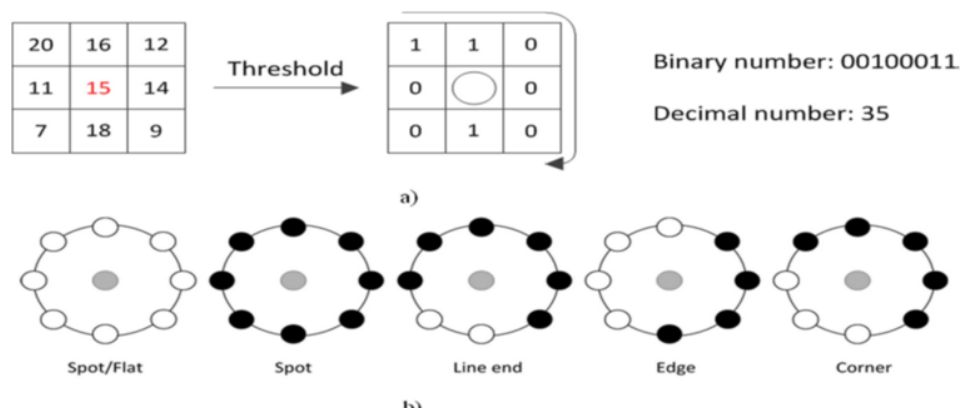

Figure 1. (a) LBP operator. (b) Texture primitive examples.

For the pixel $(\mathrm{x}, \mathrm{y})$ the LBP tag is given as

$$
\operatorname{LBP}_{(x, y)}=\sum_{r=0}^{7} i\left(m_{n}-m_{c}\right) 2^{r}
$$




$$
\text { Where } I\{X\}= \begin{cases}1, & X \geq 0 \\ 0, & X<0\end{cases}
$$

Figure 1(b) indicates that the bits can be cluster to point out neighborhood primitives such as points, lines, edges, and angles (Esfahanian et al., 2013). In this, zeros indicate the black circles and ones indicate the white circle.

\subsection{Local Directional Pattern (LDP)}

The LDP descriptor is an upgraded local texture descriptor. It assigns the eight-bit binary code to each pixel of the image. It compares the qualified value of the edge responses of each pixels in different directions. The edge detectors like Kirsch, Prewitt, and Sobel can be used at this time. The Kirsch edge detector is also known more precisely to detect the different reactions of the directional edges because, it takes all the eight neighbors into the account (Lee, 1996). These masks are displayed in the figure. 2 .

$$
\begin{aligned}
& {\left[\begin{array}{ccc}
-3 & -3 & 5 \\
-3 & 0 & 5 \\
-3 & -3 & 5
\end{array}\right] \quad\left[\begin{array}{ccc}
-3 & 5 & 5 \\
-3 & 0 & 5 \\
-3 & -3 & -3
\end{array}\right] \quad\left[\begin{array}{ccc}
5 & 5 & 5 \\
-3 & 0 & -3 \\
-3 & -3 & -3
\end{array}\right] \quad\left[\begin{array}{ccc}
5 & 5 & -3 \\
5 & 0 & -3 \\
-3 & -3 & -3
\end{array}\right]} \\
& \text { East } M_{0} \quad \text { North East } M_{1} \quad \text { North } M_{2} \quad \text { North West } M_{3} \\
& {\left[\begin{array}{ccc}
5 & -3 & -3 \\
5 & 0 & -3 \\
5 & -3 & -3
\end{array}\right]\left[\begin{array}{ccc}
-3 & -3 & -3 \\
5 & 0 & -3 \\
5 & 5 & -3
\end{array}\right] \quad\left[\begin{array}{ccc}
-3 & -3 & -3 \\
-3 & 0 & -3 \\
5 & 5 & 5
\end{array}\right] \quad\left[\begin{array}{ccc}
-3 & -3 & -3 \\
-3 & 0 & 5 \\
-3 & 5 & 5
\end{array}\right]} \\
& \text { West } M_{4} \quad \text { South West } M_{5} \quad \text { South } M_{6} \quad \text { South East } M_{7}
\end{aligned}
$$

Figure 2. Kirsch masks

Provided the center pixel of the image, the eight values of the directed edge section $\left\{\mathrm{m}_{\mathrm{i}}\right\}, \mathrm{i}=0,1, \ldots, 7$ are calculated by Kirsch mi masks in eight diverse directions aligned on its center. The occurrence of an angle or an edge is an important response value in certain directions. Therefore to generate LDP, knowing the directions is most important. Here, upper $\mathrm{k}$ direction responses would be fixed to 1 . The lasting bits $(8 \mathrm{k})$ of 8 bits of the LDP form is fixed to 0. Finally, from the formula (3) LDP code is obtained. Figure. 3 displays the mask response and the position of the LDP bit. Figure 4 explains the LDP code with $\mathrm{k}=3$.

$$
L D P_{k}=\sum_{i=0}^{7} b_{i}\left(m_{i}-m_{k}\right) \times 2^{i}
$$

Where $\mathrm{k}^{\text {th }}$ most significant response is represented as $\mathrm{m}_{\mathrm{k}}$.

$$
b_{i}(a)= \begin{cases}0, & a<0 \\ 1, & a \geq 0\end{cases}
$$



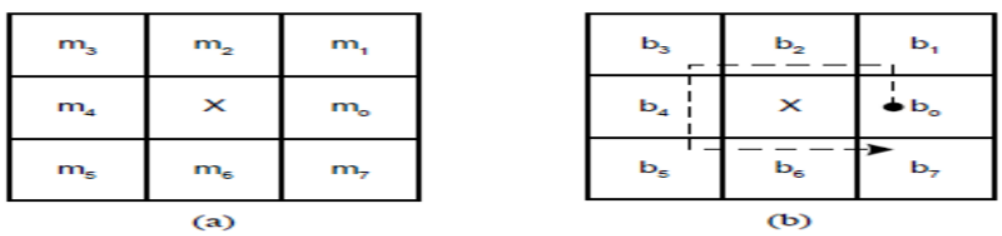

Figure 3. (a) Edge response positions. (b) LDP binary bit directions.

\begin{tabular}{|c|c|c|c|c|c|c|c|c|c|}
\hline 85 & 32 & 26 & \multirow{2}{*}{$\mathrm{M}_{\}}$} & 313 & 97 & 503 & 0 & 0 & 1 \\
\hline 53 & 50 & 10 & & 537 & $x$ & 393 & 1 & $x$ & 1 \\
\hline 60 & 38 & 45 & & 161 & 97 & 161 & 0 & 0 & 0 \\
\hline
\end{tabular}

Figure 4. LDP code.

\subsection{Local Optimal Oriented Pattern (LOOP)}

The LOOP descriptor is the inclusion of LBP and LDP (Chakraborti et al., 2018). Preference is given to the Local Optimal Oriented Pattern to overthrow the disadvantages of the two LBP and LDP.We use the Kirsch mask for coding, which is used in LDP. These forms are attached by determining the weights of each binary optimization of the pixel that relates to the kirsch result in the particular direction of the pixel. The value of $\operatorname{LOOP}\left(\mathrm{P}_{\mathrm{C}}, \mathrm{K}_{\mathrm{C}}\right)$ for a single pixel is specified by

$$
\begin{aligned}
& \operatorname{LOOP}_{\left(p_{c}, q_{c}\right)}=\sum i\left(a_{n}-a_{c}\right) \cdot 2_{n}^{w} \\
& \text { Where } i(X)=\left\{\begin{array}{l}
1, \quad x \geq 0 \\
0, \text { otherwise }
\end{array}\right.
\end{aligned}
$$

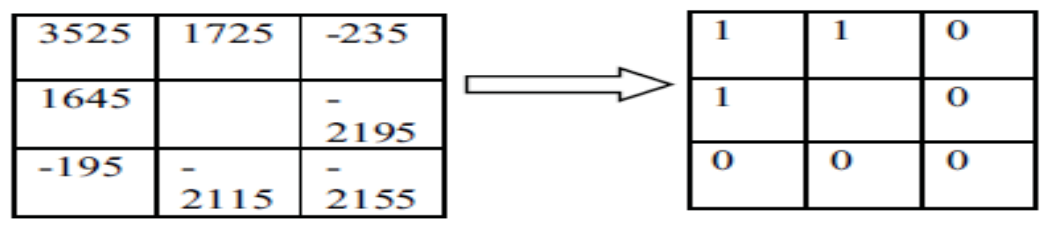

Figure 5. LOOP Calculation

According to the image, the value $\mathrm{k}$ is determined in decreasing order. Consequently, the higher values for the first three degrees the values will be fixed to 1 , other values related to 0 .

The LOOP value is calculated as: $2^{7}+2^{6}+2^{5}+0=224$ 
Thus, the LOOP pattern codes rotational invariance in the predominant wording. Also, a cycle is proposed. This method overrides the positioning of the value of $\mathrm{k}$ in the conventional LDP pattern. Figure 5 illustrates the LOOP calculation.

\section{Classification of Ovarian cyst}

In medical image processing, Feature extraction plays an significant role because it extracts the most essential information from unprocessed data which is accurate for characterization purposes while minimalizing the variations and improvements within classes (Kumar \& Bhatia, 2014). Based on the application, it is necessary to select the appropriate feature extraction algorithm to classify the ovarian cyst exactly. In this paper, the set of 100 ovarian cyst ultrasound images was used which includes 50 benign and 50 malignant cysts. Initially, the input images are denoised to improve quality. In the ovarian ultrasound image, automatic segmentation becomes very difficult as the ROI and background share the same grayscale values. Therefore, and ROI (which is an ovarian cyst in our study) is segmented manually. The segmented images are given to various textural descriptors such as LBP, LDP, and LOOP to extract features like mean and standard deviation (Priya \& Banu, 2012). Using these features, the accuracy of the classifiers is calculated and compared. The overall process is displayed in Figure 6.

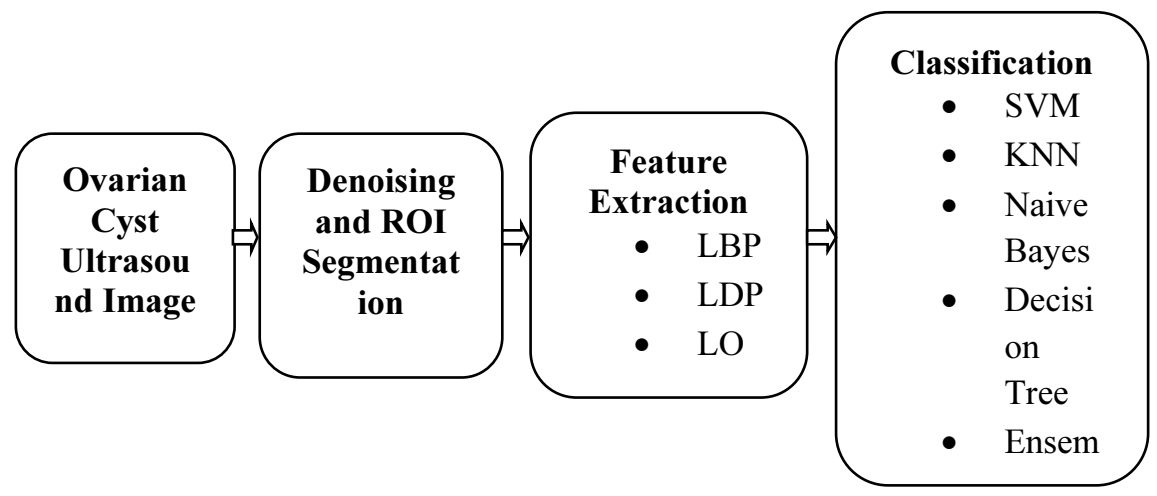

Figure 6. The process of ovarian cyst classification

\section{Results and Discussion}

The experiments are performed on Dell PC Intel(R) core ${ }^{\mathrm{TM}}$ i3 $2.30 \mathrm{GHz}$ processing speed and 4.0 GB RAM. We used the MATLAB 2019 software on windows 10 operating system. The performance of various textural descriptors was studied using statistical measures like mean and standard deviations. The chart for comparing the accuracies of classifiers is displayed in Figure 7. 


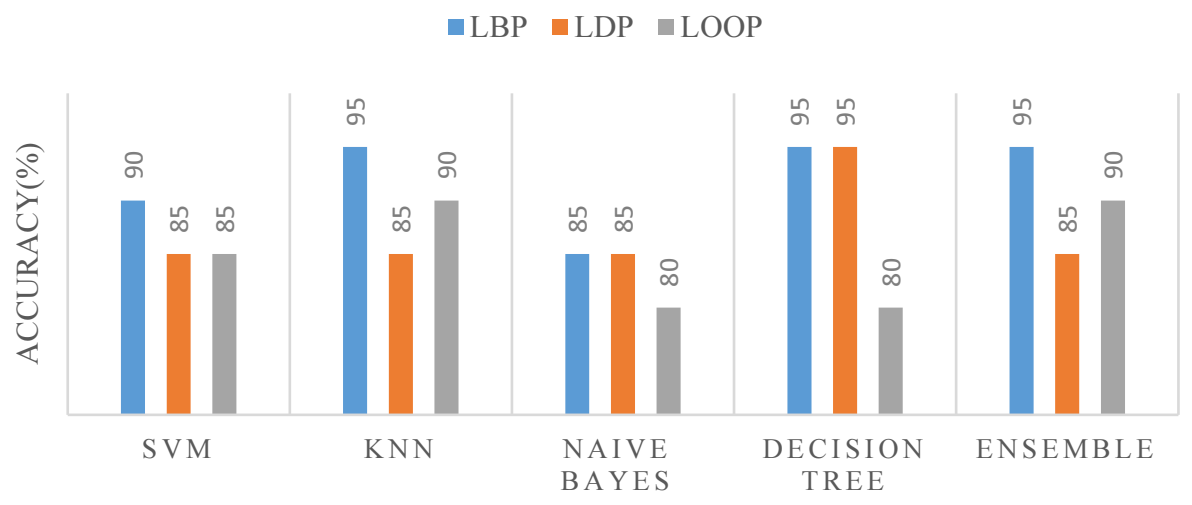

CLASSIFIERS

Figure 7. Performance Comparison.

\section{Conclusion}

In the ovarian ultrasound images, as the ROI and background share the same grayscale values, it is very difficult for the physician to classify the ovarian cyst as a simple cyst or cancerous cyst. In this paper, various textural descriptors are reviewed and two main features like mean and standard deviations are extracted using these descriptors. Finally, based on the extracted features, classifiers are used to classify the ovarian cyst. From this study, it is concluded that the Local Binary Pattern (LBP) is a suitable texture feature extraction technique for the classification of ovarian cyst than the other textural descriptors.

\section{Reference}

[1] Bala, R. (2017). Survey on Texture Feature Extraction Methods. 7(4), 10375-10377.

[2] Chakraborti, T., McCane, B., Mills, S., \& Pal, U. (2018). LOOP Descriptor: Local Optimal-Oriented Pattern. IEEE Signal Processing Letters, 25(5), 635-639. https://doi.org/10.1109/LSP.2018.2817176

[3] Esfahanian, M., Zhuang, H., \& Erdol, N. (2013). Using local binary patterns as features for classification of dolphin calls. 134(July), 105-112.

[4] George, M., \& Zwiggelaar, R. (2019). Comparative study on local binary patterns for mammographic density and risk scoring $\uparrow$. Journal of Imaging, 5(2). https://doi.org/10.3390/jimaging5020024

[5] Huang, D., Shan, C., Ardabilian, M., Wang, Y., \& Chen, L. (2011). Local binary patterns and its application to facial image analysis: A survey. IEEE Transactions on Systems, Man and Cybernetics Part C: Applications and Reviews, 41(6), 765-781. https://doi.org/10.1109/TSMCC.2011.2118750

[6] Jabid, T., Kabir, M. H., \& Chae, O. (2010a). Gender classification using the local directional pattern (LDP). Proceedings - International Conference on Pattern Recognition, 2162-2165. https://doi.org/10.1109/ICPR.2010.373

[7] Jabid, T., Kabir, M. H., \& Chae, O. (2010b). Local Directional Pattern (LDP) - A robust image descriptor for object recognition. Proceedings - IEEE International Conference on Advanced Video and Signal Based Surveillance, AVSS 2010, 482-487. https://doi.org/10.1109/AVSS.2010.17

[8] Kumar, G. (2014). A Detailed Review of Feature Extraction in Image Processing Systems. March. https://doi.org/10.1109/ACCT.2014.74 
[9] Kumar, G., \& Bhatia, P. K. (2014). A detailed review of feature extraction in image processing systems. International Conference on Advanced Computing and Communication Technologies, ACCT, 5-12. https://doi.org/10.1109/ACCT.2014.74

[10] Lee, S. W. (1996). Off-line recognition of totally unconstrained handwritten numerals using a multilayer cluster neural network. IEEE Transactions on Pattern Analysis and Machine Intelligence, 18(6), 648-652. https://doi.org/10.1109/34.506416

[11] Mattivi, R., \& Shao, L. (2009). Human action recognition using LBP-TOP as sparse Spatio-temporal feature descriptor. Lecture Notes in Computer Science (Including Subseries Lecture Notes in Artificial Intelligence and Lecture Notes in Bioinformatics), 5702 LNCS, 740-747. https://doi.org/10.1007/9783-642-03767-2 90

[12] Narain Ponraj, D., Christy, E., Aneesha, G., Susmitha, G., \& Sharu, M. (2019). Analysis of LBP and LOOP Based Textural Feature Extraction for the Classification of CT Lung Images. Proceedings of the 4th International Conference on Devices, Circuits and Systems, ICDCS 2018, 309-312. https://doi.org/10.1109/ICDCSyst.2018.8605138

[13] Ojala, T., Pietikäinen, M., \& Harwood, D. (1996). A comparative study of texture measures with classification based on feature distributions. Pattern Recognition, 29(1), 51-59. https://doi.org/ 10.1016/0031-3203(95)00067-4

[14] Ojala, T., Pietikäinen, M., \& Mäenpää, T. (2002). Multiresolution gray-scale and rotation invariant texture classification with local binary patterns. IEEE Transactions on Pattern Analysis and Machine Intelligence, 24(7), 971-987. https://doi.org/10.1109/TPAMI.2002.1017623

[15] Oppedal, K., Eftestøl, T., Engan, K., Beyer, M. K., \& Aarsland, D. (2015). Classifying dementia using local binary patterns from different regions in magnetic resonance images. International Journal of Biomedical Imaging, 2015. https://doi.org/10.1155/2015/572567

[16] Zafar, M. Z., Adnan, S. M., Ahmad, W., Ikram, J., \& Rashid, J. (2019). Brain Tumor Detection and Classification Using Geometrical Shapes as Texture Descriptors. May.

[17] V.D.Ambeth Kumar, Dr.S.Malathi, V.D.Ashok Kumar (2015) "Performance Improvement Using an Automation System for Segmentation of Multiple Parametric Features Based on Human Footprint” for the Journal of Electrical Engineering \& Technology (JEET), vol. 10, no. 4, pp.1815-1821 , 2015.

[18] V.D. AmbethKumar, V.D.AshokKumar,G. KumuthaRajeswari, M.Anitha , "Human Character Identification Based On a New Biometric Pattern - A Contemporary Approach", Volume 133, Pages 99-107, Procedia Computer Science (Elsevier), 2018. 